\title{
Study of the public service satisfaction degree in China city
}

\author{
Chen Fan, Wang Shengjin* \\ Shanghai Urban Construction Vocational College, Shanghai, 201415, China
}

\begin{abstract}
The measurement of public service satisfaction involves many latent variables and the validation of a causal model, and the application of structural equation modelling can effectively solve these problems. This study constructs a customer satisfaction index model for measuring the satisfaction degree of public service in China City. The main factors and its loading coefficients of affecting the public service quality of China City are verified; what's more, the suggestions on improving the satisfaction degree of public service quality are proposed.
\end{abstract}

\section{Introduction}

The public service satisfaction appraisal involves the measurement of many latent variables and the verification of the causal model, and the application of the structural equation model can effectively solve the problems. The paper first briefly introduces the methods of structural equation model, then constructs the customer satisfaction index model to measure the satisfaction degree of China city public service department, finally verifies the main factors and load coefficient affecting it and puts forward suggestions about methods of improving public service satisfaction degree.

It is necessary to further develop the methods because of many problems in the existing appraisal methods. The structural equation modelling developed in recent years provides an effective tool for government satisfaction appraisal.

\section{Research methods: SEM}

Many assumptions constructs cannot be directly measured or observed in the social behaviour science, and this assumption is only one characteristic constructs or abstract concept which cannot be learned directly. So, the construct characteristics can only be reflected directly by some scales or by the actual observed data.

Structural equation modelling (SEM) is also called by some scholars as the latent variable model (LVM) (Moustaki, Jöreskog et al. 2004). SEM is basically a confirmatory analytical method, which must be supported by theory or experience rules and guided by theory. SEM is a mathematical model of rendering the objective state, which is mainly used to test the hypothesized relationship between observed variables and latent variables (endogenous variables and exogenous variables). It combines two statistical techniques: factor analysis and path analysis.
There are two basic models in SEM: measured model and structural model. The measured model is made up of latent variables and observed variables. Observed variables are mainly the data obtained by the scale or questionnaire measurement tools; latent variable is characters or abstractions formed among observed variables. The characters or abstractions cannot be directly measured, but it can be by reflected by the data of observed variables. Observed variables are usually shown as rectangular symbols and latent variable as oval-shaped symbol in SEM.

In SEM, a latent variable must be estimated by more than two observed variables, which is called the principle of multi-index. Latent variable can just totally explain the measure index if there is only one measured variable for each latent variable, and the error is 0 . The regression equation of measured model is:

$$
\begin{gathered}
X=l_{x} x+d \\
Y=l_{y} h+e
\end{gathered}
$$

Structural model refers to the instructions of the causal relationship of latent variable models. As a cause, the latent variable (also called as endogenous variable) is often expressed in symbols $\xi$; As a result, the latent variable (also called as exogenous variable) is often expressed in symbols $\eta$. The structural model equation can be expressed in matrix as:

$$
h=b h+\mathrm{Gr}+z
$$

In the SEM analysis, the relationship between variables is very complex because of it involving many measuring models and a structural model. Therefore, the establishment of the relationship between variables must have a strong theoretical basis. At the same time, the model's definition should follow the principle of

e-mail: CJ0840@succ.edu.cn 
parsimony. According to the principle of parsimony, theoretical basis is needed when each parameter is defined. A relationship without theoretical support or weak theory should be excluded out of the hypothesis model (Fangming Huang, 2005).

\section{The application of SEM in public service appraisal assessment}

There are many appraisal models to evaluate the public satisfaction degree about public departments, such as the American Customer Satisfaction Index (ACSI), the Europe Customer Satisfaction Index (ECSI), and the Sweden's Customer Satisfaction Index (SCSB) and so on. But the customer satisfaction appraisal in our public departments is still at the beginning. Domestic scholars' researches mainly focus on how to construct or import the customer satisfaction index model. Empirical studies on the public satisfaction degree about public departments are very rare. Meanwhile, public satisfaction in public departments is different from that in the enterprise management. It involves broader aspects, and requires more targeted index systems and models. Therefore, we need to explore appropriate appraisal models to measure the public satisfaction degree of China city.

The author made the public service satisfaction survey from 2009 to 2010. In 2009, the survey is made in the administrative service centre in six districts of China for public service satisfaction assessment. The evaluation object is a single public sector; this study classifies various public services into seven categories, which include administration, justice, life service, education, media, and community, ruling and defence sectors. 32 public service institutions which are related to residents' daily life are selected. According to the two different evaluation objects, the author constructs different customer satisfaction index models.

\subsection{The index model of public service satisfaction in the administrative service centre}

The administrative service centre is a new model of local government public service. The government brings together most of the window units which directly serve the citizens. People can handle a variety of examination and approval items, which can reduce the cost of service. Conducting the survey on citizens who have just left the administrative service centre, this research can accurately measure the customer satisfaction of the local government. It is divided into several stages such as model building, research hypotheses, questionnaire design, questionnaire survey and results analysis.

\subsection{The construction of public service satisfaction index}

Research model A uses the main structure variables of American public sector customer satisfaction index model: customer expectation, perceived quality, customer satisfaction, complaints and loyalty. It has made some improvements based on the features of our government's public service. According to the characteristics of the government service; the microscopic quality measurement is introduced. Thus, we can not only get the whole quality data but also clearly know microscopic quality information.

The observed variables for latent variables of customer complaints are adjusted by this research. Most of the public thinks the government is a symbol of authority under the influence of political culture. Public complaints due to dissatisfaction with the government service are few. Although there is more and more public participation in the government public service evaluation, the influence of traditional culture and the government complaint mechanism still bound the public to choose complaints when they want to express the dissatisfaction with government public service. So, we will not ask if they ever held complaints about government departments in designing observation variables, but in some ways such as their complaining ideas, acquaintances complaints and strange complaints, which to some degree can reflect different degrees of government complaints and more accord with national conditions.

Observed variable of latent variables-customer loyalty has also been adjusted. "Whether you are going to move out of the city" or "whether you trust the government" is used by American Customer Satisfaction Index model as the observation variables for customer loyalty. Considering the conditions of urban population flow are different, two observed variables, loyalty and government purpose, replace the American variables.

\subsection{Questionnaires and surveys}

As to the requirements of samples number, Ding et al. (Ding, Velicer et al. 1995) suggested that the minimum number of test samples is 100-150 when covariance structure modelling is used. Boomsma (Boomsma.1987) proposed that the minimum sample size is 200 when maximum likelihood method is used to estimate the structural equation modelling. Erroneous inference results will be led if the sample number is less than 100. Marsh et al. (Marsh, Hua et al. 1998) pointed out that the more indicator variables of latent variables is, the model estimation result will be better from the perspective of the model convergence degree, the stability of the parameters and the reliability of theoretical construction.

The author selected six administrative service centres for investigation in China City. The objects of the questionnaire are the residents of the six centres in China. The random sampling survey method is adopted. Thirty residents who have just finished their matters are asked for interviews in every administrative service centre. The questionnaire survey uses blank-filling forms. 500 copies were sent out and 418 copies were returned, among which 383 are valid. The valid ratio was $91.63 \%$. Analysis showed the Cronbach's coefficient of the questionnaire is 0.817 , which indicates that the reliability can be accepted. 
4 Model fitting

4.1 Analysis of the model fit result
The data are processed in AMOS7, and the main fitness index is as Table 1.

Table1. Index of model fit goodness

\begin{tabular}{cc}
\hline Index & Default model fitness \\
\hline 2 & 214.66 \\
DF & 122 \\
RMSEA & 0.038 \\
NFI & 0.93 \\
CFI & 0.91 \\
AGFI & 0.92 \\
\hline
\end{tabular}

The value of $\chi 2 / \mathrm{DF}<2$ and $\mathrm{RMSEA}=0.038$, and the other index has also reached the acceptable level. So, the hypothesized model can be used to measure the satisfactory degree of local government service quality.

\section{Research conclusions}

\subsection{Perceived quality has the biggest influence on public satisfaction with a direct effect of 0.69 , whereas its direct effect on public trust is 0.06 .}

This shows that perceived quality has a big direct positive influence on public satisfaction, but the influence of public trust is weak. This phenomenon is interesting, which means that how the public feel about public service has a greater influence on satisfaction than trust.

\subsection{The organization image has a great} influence on perceived quality with the direct effect of 0.82 , the direct positive effect on public satisfaction and public trust are 0.20 and 0.23 .

The perceived quality of the public is directly influenced by the image of public service departments. Perhaps it is because if the public has a better impression on public departments, they will more forgivable towards mistakes and more affirmative towards achievements, thus the perceived quality is raised. But why the image has a weak influence on public satisfaction and public trust? This is because satisfaction is mainly delivered by perceived quality, whereas organization image is only an indirect factor, which reflects the overall impression of the public departments, rather not the specific impression of certain department.

\subsection{The public satisfaction has the biggest influence on public trust with the direct effect of 0.90 .}

This shows that public satisfaction in China city can directly lead to the public support for public service departments. It illustrates from the above results that public department of China city needs to further improve its service functions and raise the satisfaction degree of the public to stimulate and win the trust of the public.

\section{References}

1. Savalei, V., \& Bentler, P. M. (2010). Structural equation modeling. Corsini Encyclopedia of Psychology., 13:161-182.

2. Bielby, W. T., \& Hauser, R. M. (1977). Structural equation models.Annual Review of Sociology., 3(1):137-161.

3. Anderson, J. C., \& Gerbing, D. W. (1988). Structural equation modeling in practice: $\mathrm{A}$ review and recommended two-step approach. Psychological Bulletin, 103(3): 41-67.

4. Wu, R. Z., Zhai, D. D., Xi, E. C., \& Li, L. (2009). Evaluation model of satisfaction degree for urban public transit service.Journal of Traffic and Transportation Engineering., 9(4):65-70. 